\title{
LA CULTURA ORGANIZACIONAL: ABORDAJE CONCEPTUAL Y DELIMITACIÓN DE VARIABLES PARA SU ESTUDIO.
}

\section{ORGANIZATIONAL CULTURE: CONCEPTUAL APPROACH AND DELIMITATION OF VARIABLES FOR YOUR STUDY.}

\author{
Mónica del Pilar Llanos Encalada, Mgs. \\ Magíster en docencia universitaria e investigación educativa (Ecuador). \\ Asesora en Gestión del Talento Humano- Psicología laboral. \\ Docente Titular de la Facultad de Ciencias Económicas y Empresariales de la \\ Universidad Tecnológica ECOTEC, Ecuador. \\ mllanos@ecotec.edu.ec
}

Rosa Elis Bell Heredia, PhD. Doctora en Economía (Cuba). Presidenta - Sección de Economía - Comisión Nacional de Grados. Profesora del Departamento de Ciencias Empresariales de la Facultad de Economía de la Universidad de La Habana, Cuba. rosabellp@gmail.com

\section{ARTÍCULO DE REFLEXIÓN}

Recibido: 8 de febrero de 2018

Aceptado: 22 de marzo de 2018

\section{RESUMEN}

En la actualidad las organizaciones se enfrentan a constantes retos en correspondencia con las expectativas del mundo globalizado. Esto lleva al desarrollo de las organizaciones y las relaciones laborales, siendo el personal la clave del éxito por encontrarse detrás de todo proceso de gestión. Al valorarar los intangibles relacionados con las buenas prácticas en la gestión del talento humano, la situación se vuelve compleja, puesto que las variables que las componen (cultura; clima; calidad de vida laboral), resultan imprecisas al momento de su estudio. Por lo cual, el objetivo del presente estudio es esclarecer el concepto de cultura organizacional y delimitar variables objetivas que permitan su valoración al interior de las organizaciones, por ello 
se analizan los aportes teóricos de 18 autores representativos en el tema, desde la década del 70 hasta la actualidad.

Palabras clave: cultura organizacional, gestión del talento humano.

\section{ABSTRACT}

Today organizations are faced with constant challenges to achieve success regarding the expectations of a globalized world. This contributes to the development of the organizations and their labor relations, whose personnel are the key to success because they work behind the scenes in every management process. When valuing the intangibles related to good practices in the management of human talent, the situation becomes complex, since the variables that compose them (culture, climate, quality of work life), are imprecise at the time of their study. Therefore, the goal of the present study is to clarify the concept of organizational culture and to delimit objective variables that allow its assessment within organizations, for it, the theoretical contributions of 18 representative authors in the subject are analyzed, from the 70 s to the present .

Keywords: management human talent; organizational culture.

\section{INTRODUCCIÓN}

Las organizaciones han evolucionado, no solo en su estructura y forma de administrarse, sino también en la manera de concebir y gestionar a su personal. Está presente la dinámica de las relaciones interpersonales y los aspectos psicológicos en las personas que influyen en su desempeño laboral, su identidad y su involucramiento con los objetivos organizacionales.

Con el paso de los años, se ha dado un cambio de paradigmas que obliga a insertar principios humanistas en las organizaciones gracias a los aportes de la psicología organizacional y social. Esto trae diferente visión en la gestión de las personas, es decir, se pasa de administración de recursos humanos, a gestión del talento humano (GTH). Más allá de un cambio de conceptos, esto implica significantes de trascendencia en la acción y la gestión, entre ellos, dando importancia a intangibles como la cultura organizacional.

La cultura se refleja en el comportamiento de sus miembros y en la imagen que proyecta la organización a lo interno y externo. Desconocer su importancia trae como 
consecuencia culturas débiles y en muchas ocasiones contrarias a los propósitos que persiguen las organizaciones. La cultura organizacional permite identificar y diferenciar unas organizaciones de otras.

Los primeros estudios sobre cultura organizacional, surgen en los años setenta, con los aportes de Pettigrew (1979) y Dandridge, Mitroff y Joyce (1980). A partir de allí, surgen otros autores representativos como Geert Hofstede (1991); Sthepen Robbins (2013); Idalberto Chiavenato (2009); Edgar Schein (1986-2010); Martha Alles (2007), entre otros.

De la literatura consultada, se ha seleccionado el aporte de 18 autores que estudian la cultura, algunos de ellos de manera superficial y uno pocos que amplían su estudio. Luego del análisis de estas aportaciones, las autoras delimitan la cultura en dos dimensiones de las cuales se desagregan seis variables, lo cual va a permitr contar con aspectos objetivos que permitan la valoración de la cultura.

\section{REVISIÓN TEÓRICA}

Uno de los primeros autores en estudiar la cultura organizacional es Pettigrew (1979),citado por (Cújar, Ramos, Hellman, \& Jorge, 2013) quien la considera como "El sistema de significados públicamente y colectivamente aceptados operando para un grupo determinado en un tiempo dado". Tal concepto influye en Dandridge, Mitroff y Joyce (1980), quienes introducen el término "simbolismo organizacional", en referencia a los varios usos simbólicos. (Cújar, Ramos, \& Jorge, 2013). Para ellos, la cultura organizacional se debe estudiar a partir de la revelación profunda de varios aspectos.

Posteriormente, Schwartz y Davis (1981), afirman que la cultura organizacional es "un patrón de las creencias y expectativas compartidas por los miembros de la organización. Estas creencias y expectativas producen normas que que regulan la conducta de los individuos y los grupos en la organización" (Schein,1987). Este concepto fue ampliado, años más tarde, por Barney (1986), para quien la cultura organizacional puede convertirse en una ventaja competitiva (Schein, 1987). Para Becker (1998), citado por Robbins (2013), la cultura organizacional se refiere a un sistema de significados compartidos por los miembros de una organización. 
Uno de los autores que amplía el concepto de cultura organizacional es Edgar Schein (1986-1987). En sus primeros escritos, parte del análisis de las personas y las organizaciones como entes vivos y dinámicos, posteriormente hace aportes muy valiosos en cuanto a la influencia del liderazgo. En uno de sus escritos, identifica a la cultura, como "el nivel más profundo de presunciones básicas y creencias que comparten los miembros de una empresa y lo hacen de una manera inconsciente, lo cual les permite definir y tener una interpretación básica de la imagen que la empresa tiene de sí misma y del entorno".(Schein 1986)

Para Schein (1987), la cultura se presenta en tres niveles: "1) el de los artefactos, referido a lo que se observa en las personas: forma de vestir, manera de hablar y comportarse; 2) los valores compartidos y asumidos por la mayoría de miembros en la organización y 3) los supuestos básicos que constituyen los niveles más íntimos, profundos y ocultos, esto referido a: las creencias, percepciones, sentimientos y supuestos dominantes en los que las personas creen". (Schein, 1987).

Schein (1987), define el liderazgo a partir de cinco características básicas que definen el estilo de liderazgo en las organizaciones: “1) Percepción de los líderes en cuanto a la relación que tiene la institución con el entorno (dominar y cambiar; someterse y asumir lo impuesto; coexistir y estar en armonía); 2) distribución de tareas de sus equipos de trabajo y manejo del tiempo, lo que se traduce en trabajo monocrónico (una tarea a la vez); o policrónico (varias tareas a la vez). Los horarios; puntualidad o no; tiempo dedicado a reuniones; 3 ) percepción de la naturaleza humana: (buenos, malos, neutros; perfectibles o no; 4) percepción sobre la naturaleza de la actividad (activospasivos;optimistas- pesimistas;innovadores-conservadores;controlados-manipulados); 5) naturaleza de las relaciones: distribución del poder (competitiva-cooperativacompetente; individual-asociativa) y cumplimiento de tareas y objetivos impulsado por (ley-autoridad o carisma) ". (Schein 1987)

Schein (1987), refiere que para Martin y Siehl (1983) la cultura de una organización puede sufrir cambios espontáneos, ya sean intencionados por la gerencia, o por causa de las subculturas, lo cual puede ayudar a reforzar la cultura principal.

Hofstede (1991), inicia sus estudios de cultura con relación a los diferentes países y ámbitos de interculturalidad que se dan en diferentes escenarios. Posteriormente se centra y profundiza en el ámbito organizacional y la relación de la cultura con la 
dirección. Realiza una investigación muy valiosa en la empresa multinacional IBM. "En contraste, con la mayoría de estudios organizacionales anteriores, que incluían un número limitado de países o diferentes compañías en distintos países...Hofstede encuesta a más de 116000 empleados de 40 países..." (Quiroz \& Valtierrez, 2010).

Enfatiza que si las personas sienten; piensan y actúan de diferentes maneras, es gracias a la cultura. Afirma además que estructura de maneras diferentes la mente de las personas. Para explicarlo adopta el término "el software de la mente", ya que la cultura programa mentalmente a las personas. "La programación empieza con la familia, luego continúa con el vecindario, la escuela, en los grupos que una persona tiene, en los lugares de trabajo y en la vida de comunidad" (Hofstede, 1991). Por lo tanto la cultura se va a construir a partir de lo que las personas son y el resultado de sus interrelaciones.

Se entiende que la cultura no es algo inherente al ser humano, es algo aprendido, como consecuencia del desarrollo social y no de los genes. Por ello, la cultura se sustenta en cuatro áreas: "Valores; rituales; héroes y símbolos, los cuales se expresan a través de las prácticas" Hofstede (1991). Además, se hace una clara diferencia entre características comunes a todas las personas, de aquellas que son adquiridas a través de los años por la cultura; $y$, la personalidad de cada individuo.

Años más tarde Hofstede (2002), identifica cuatro tipos de cultura que se pueden dar en las organizaciones: "distanciamiento del poder; aceptación del nivel de incertidumbre; individualismo y masculinidad", establecidas en cuanto a su nivel de desarrollo entre baja; mediana y alta. Plantea, además, que toda organización independientemente del sector en el que opere, debe desarrollar una cultura local.

Autores reconocidos por el establecimiento de ítems de valoración de la cultura, Cameron \& Quinn (1999), citado por (Salazar A. M., 2008), proponen un modelo de evaluación basándose en los "esquemas categóricos conocidos y aceptados que organizan el modo en que la gente piensa, sus valores y suposiciones y la manera como procesan la información" (Salazar A. M., 2008). Su modelo parte del principio de categorizar a la cultura organizacional en cuatro tipos: "Clan; Adhocracia; Jerarquizada y Mercado".

Las autoras no comparten las categorías establecidas por Hofstede (2002) y Cameron \& Quinn (1999), pues consideran que la cultura de cada organización tiene 
características propias que la identifican y diferencian de otras, aun cuando puedan coincidentemente tener características de una u otra de las categorías mencionadas por dichos autores.

Para Robbins \& Judge (2013), la cultura organizacional representa un grado de percepción sobre la organización, que suele ser común a todos sus miembros. Sin embargo, pueden existir sub culturas al interior de la organización. La postura de Robbins (2013), se basa en las investigaciones realizadas por G. Hofstede, B. Neuijen, D. Ohayv, y G. Sanders (1991), a partir de lo cual define la cultura organizacional con diez características básicas.

Los autores citados resaltan como elementos de la cultura: los límites; la identidad; el compromiso; la estabilidad del sistema social, lo cual ayuda a mantener unida la organización; así como el establecimiento de normas apropiadas que hacen las veces de mecanismos de control que modelan el comportamiento y las actitudes de las personas. Sin embargo, para estos conceptos resultan amplios para el estudio de la cultura.

Para, Cruz (2001), citada por González (2012), plantea una definición que no difiere mucho de los autores mencionados. "Es una forma aprendida de hacer... que se comparte por sus miembros, consta de un sistema de valores y creencias básicas que se manifiesta en: normas, actitudes, conductas, comportamientos, la manera de comunicarse, las relaciones interpersonales, el estilo de liderazgo, la historia compartida, el modo de dar cumplimiento a la misión y la materialización de la visión, en su interacción con el entorno, en un tiempo dado".

Chiavenato (2009), desarrolla la teoría de la gestión del talento humano y como parte de esta, la cultura organizacional. Para él la cultura representa el conjunto de normas no escritas o informales, los significados que dan las personas de la organización a su comportamiento y por lo tanto a sus prácticas. Realiza una representación gráfica de la misma mediante un iceberg en el cual la punta, que se puede observar, se encuentra representada por: las normas; políticas; misión; objetivos; procedimientos. La base, aquella que no se ve, estaría estructurada por: percepciones; sentimientos; actitudes; valores; interacciones informales; normas de grupo y otras. 
Figura 1. El iceberg en la cultura organizacional.

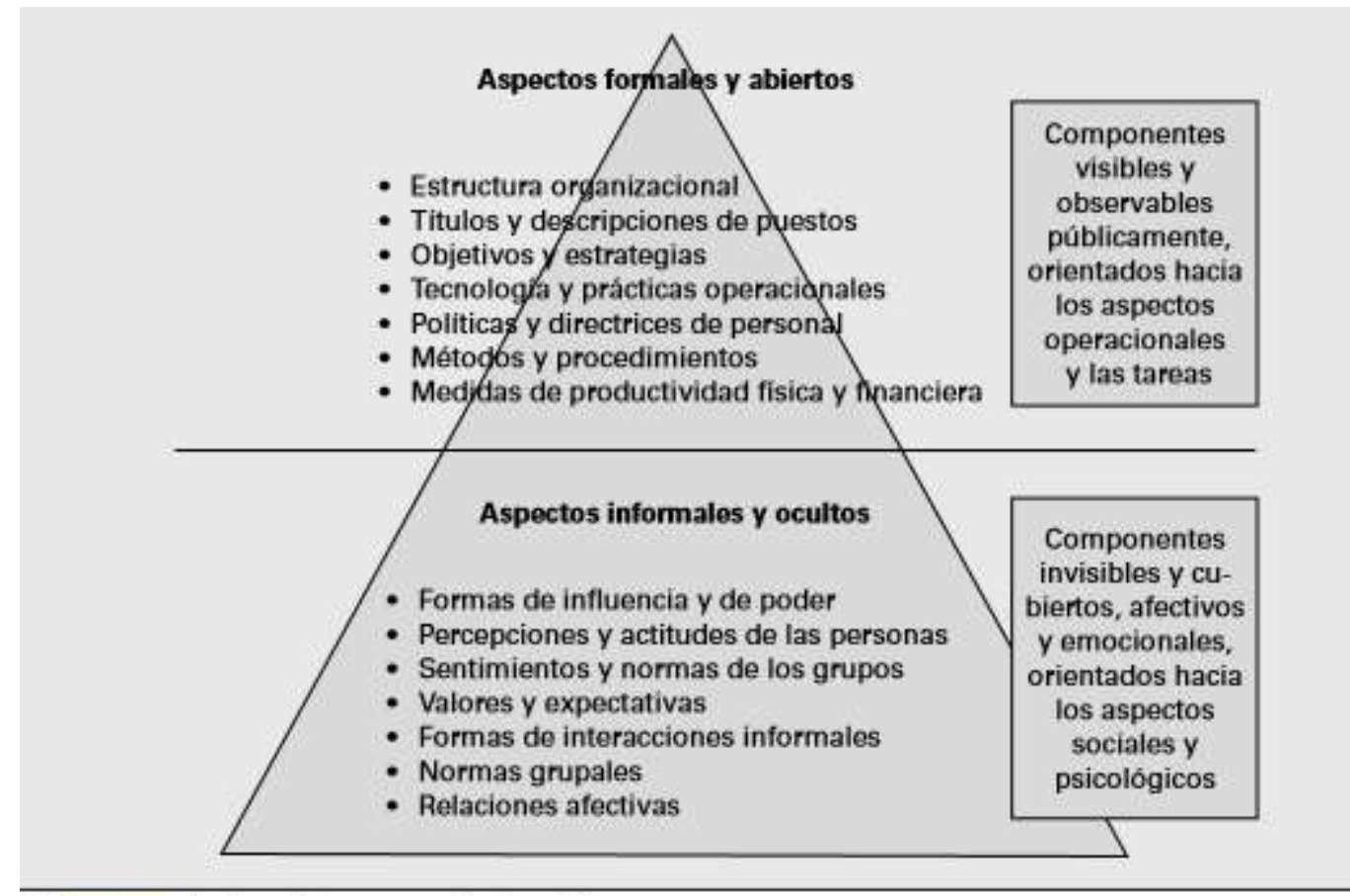

El iceberg de la cultura organizacional. ${ }^{6}$

Fuente: Chiavenato (2009)

Cuesta (2005) define a la cultura organizacional como "el conjunto de normas, valores, creencias y pautas compartidas que caracterizan los modos de hacer y comportarse de los miembros de la organización, y que determinan la forma en que se resuelven los problemas y se toman las decisiones en la organización".

Para las autoras, la cultura subyace al comportamiento organizacional y concuerdan con Cuesta en el sentido de que la cultura se evidencia en la forma de actuar de los líderes frente a la resolución de problemas y la toma de decisiones. Enfatiza además la importancia que tienen los valores como parte de la cultura, para él son "Las convicciones que sostienen el estilo de dirigir de la organización, su relación con los trabajadores y entidades externas y su ética. Son pautas del comportamiento de los trabajadores. Son el conjunto de principios morales, de preferencias culturales y actitudes lógicas que estructuran los juicios de las personas y guían su comportamiento en la organización" (Cuesta, 2005). 
La cultura organizacional para Alles (2007), es el conjunto de supuestos, convicciones y normas que comparten los miembros de una organización, esta pudo haber sido creada por sus fundadores y/o líderes o haberse formado de manera espontánea a través del tiempo. Plantea, que resulta difícil cambiar la cultura organizacional de una empresa pero no imposible. Considera que las organizaciones se desarrollan en un determinado medio, en el que los individuos viven y trabajan, ese medio se conforma de un complejo sistema de leyes, valores y costumbres al cual se lo denomina cultura social o nacional.

En un estudio realizado en México, se analiza la cultura organizacional desde varias aristas: el enfoque epistemológico; psicológico; social; político; antropológico y económico. Este abordaje le permite establecer que "Las macroculturas interorganizacionales impactan e influencian todos los aspectos que inciden en la competencia y la cooperación entre las organizaciones. Los sistemas de valores y creencias compartidos por los miembros de diferentes organizaciones que forman una macrocultura, facilitan las relaciones de cooperación y coordinación interorganizacional, contribuyen a la reducción de contradicciones, conflictos y rivalidades" (Vargas, 2007).

El aporte del estudio precedente, radica en la importancia de ver la cultura organizacional en su contexto, influenciada por la macro cultura del país y de instituciones del sector al cual pertenecen las organizaciones. En la misma línea, Abravanel; Allaire; Hobbs; Firsirotu \& Poupart (2009), realizan un análisis de dos enfoques planteados por los antropólogos: el sociocultural y el de las ideas. Concluyen que la cultura organizacional debe verse como un sistema particular de símbolos, influido por la sociedad circundante; la historia de las organizaciones; sus líderes pasados; así como también por diferentes factores de contingencia.

Los enfoques mencionados permiten entender que la cultura no es estática, sino dinámica, expresada de manera diferente por cada miembro de la organización, y transformada por ellos durante el proceso de decodificación de los acontecimientos organizacionales. Ella se articula además con principios de aprendizaje cultural expresados por Schein (1986) y Hofstede (1991).

En la misma línea de pensaminto, Hill, sostiene que la cultura de un país no difiere mucho de la cultura de una organización, puesto que "la cultura se refiere a un sistema de valores y normas que comparten las personas. Los valores son ideas abstractas 
sobre lo que un grupo considera bueno, correcto y deseable: las normas significan las reglas sociales y las directrices que prescribe una conducta apropiada en situaciones particulares." (2011)

En la siguiente definición se aprecia la integración de conceptos abstractos y subjetivos con lo tangible y visible. "La cultura es la personalidad de una sociedad e incluye ideas abstractas, como valores y cuestiones éticas y objetos materiales y servicios, automóviles, ropa, comida, arte y deportes, productos de la sociedad...es la acumulación de significados, rituales, normas y tradiciones compartidos por los miembros de una organización o sociedad" (Solomon, 2013).

En la literatura consultada, también se ha encontrado la definición de cultura en términos de fuerte o sólida y débil, atribuyendo una connotación positiva a las empresas que poseen una cultura fuerte. Las autoras no consideran que esta definición sea un aporte ya que la cultura sólida o fuerte se da cuando "... es percibida desde el exterior como una organización poseedora de un cierto estilo o manera de hacer las cosas... "sólida", no necesariamente significa "buena"... puede ser "mala" (Hill, 2011).

De los autores citados, aquellos que han desarrollado ampliamente el concepto de cultura organizacional son Schein (1986-2010); Hofstede (1991-2002) y Robbins y Judge (2013). En la tabla 1, se integran los aportes sobre cultura organizacional realizados por los 18 autores referenciados. A partir de estos, se realiza el aporte conceptual asumiendo una definición de cultura y estableciendo la delimitación seis variables que permiten su valoración.

Entre las variables mayormente abordadas por los autores están: percepcionessignificados, creencias-expectativas, las cuales resultan imprecisas para valorar la cultura. Las autoras consideran pertinente relacionar estas características a aspectos que se puedan evidenciar y sean objetivas. Otras de las variables coincidentes entre los autores son: los valores; el liderazgo, las normas y las relaciones entre los miembros. 
Revista Científica ECOCIENCIA

Tabla 1. Síntesis de los aportes sobre cultura organizacional de autores consultados.

\begin{tabular}{|c|c|c|c|c|c|c|c|c|c|c|c|c|c|c|c|c|c|c|c|}
\hline \multirow[b]{2}{*}{ Variables } & \multicolumn{19}{|c|}{ Autores } \\
\hline & $\begin{array}{l}\text { Pettigrew } \\
\text { (1979) }\end{array}$ & $\begin{array}{l}\text { Dandrige; } \\
\text { Mitroff y } \\
\text { Joyce (1980) }\end{array}$ & $\begin{array}{l}\text { Schwartz } \\
\text { y Davis } \\
\text { (1981) }\end{array}$ & $\begin{array}{l}\text { Barney } \\
\text { (1986) }\end{array}$ & $\begin{array}{l}\text { Becker } \\
\text { (1998) }\end{array}$ & $\begin{array}{l}\text { Schein, } \\
\text { Edgar } \\
(1980- \\
2000) \\
\end{array}$ & $\begin{array}{l}\text { Martín } \\
\text { y Siehl } \\
(1983)\end{array}$ & $\begin{array}{l}\text { Hofstede, } \\
\text { Geert } \\
\text { (1991-2010) }\end{array}$ & $\begin{array}{l}\text { Cameron } \\
\text { y Quinn } \\
(1999)\end{array}$ & $\begin{array}{l}\text { Stephen } \\
\text { Robbins \& } \\
\text { Judge } \\
\text { (2000-2013) } \\
\end{array}$ & $\begin{array}{l}\text { Cruz } \\
\text { (2001) } \\
\end{array}$ & $\begin{array}{l}\text { Cuesta, } \\
\text { Armando } \\
(2005)\end{array}$ & $\begin{array}{l}\text { Abravanel; } \\
\text { Allaire; Hobbs; } \\
\text { Firsirotu; Poupart } \\
\text { (2009) } \\
\end{array}$ & $\begin{array}{l}\text { Idalberto } \\
\text { Chiavenato } \\
(1995-2012)\end{array}$ & $\begin{array}{l}\text { Vargas } \\
\text { José } \\
\text { (2007) }\end{array}$ & $\begin{array}{l}\text { Alles } \\
\text { Martha } \\
\text { (2007) }\end{array}$ & $\begin{array}{l}\text { Hill } \\
\text { Charles } \\
\text { (2011) }\end{array}$ & $\begin{array}{l}\text { Solomon } \\
\text { Michael } \\
\text { (2013) }\end{array}$ & $\begin{array}{l}\text { Llanos } \\
\text { Mónica } \\
\text { (2017) }\end{array}$ \\
\hline $\begin{array}{l}\text { Percepciones } \\
\text { Significados }\end{array}$ & $x$ & $x$ & & & $x$ & $x$ & & & & $x$ & & $x$ & & $x$ & & & $x$ & $\mathrm{X}$ & \\
\hline $\begin{array}{l}\text { Creencias } \\
\text { Expectativas }\end{array}$ & & & $x$ & & & $x$ & & & $x$ & & & & & $x$ & $\mathrm{x}$ & & & & \\
\hline $\begin{array}{l}\text { Normas } \\
\text { políticas }\end{array}$ & & & $x$ & & & & & & & $x$ & $\mathrm{x}$ & $x$ & & $\mathrm{x}$ & & $x$ & $x$ & $x$ & $x$ \\
\hline $\begin{array}{l}\text { Ventaja } \\
\text { Compet }\end{array}$ & & & & $x$ & & & & & & & & & & & & & & & \\
\hline Valores & & & & & & $x$ & & $x$ & $x$ & & $x$ & $x$ & & $x$ & $x$ & $x$ & $x$ & $x$ & $x$ \\
\hline Símbolos & & & & & & $x$ & & $\mathrm{x}$ & & & & & $x$ & & & & $x$ & $x$ & \\
\hline $\begin{array}{l}\text { Pensamientos } \\
\text { Sentimientos }\end{array}$ & & & & & & & & $x$ & $x$ & & & & & $x$ & & & & & \\
\hline $\begin{array}{l}\text { Liderazgo } \\
\text { Incentivos }\end{array}$ & & & & & & $\mathrm{x}$ & $x$ & $\mathrm{x}$ & & $\mathrm{x}$ & $x$ & $x$ & $x$ & & & & & & $x$ \\
\hline $\begin{array}{l}\text { Relaciones } \\
\text { Rituales }\end{array}$ & & & & & & & & $x$ & & $x$ & $x$ & $x$ & & $x$ & $x$ & & $x$ & & $x$ \\
\hline Comunicación & & & & & & & & & & $\mathrm{x}$ & $x$ & & & & & & & & $\mathrm{x}$ \\
\hline Visión Misión & & & & & & & & & & & $x$ & & & $x$ & & & & & $x$ \\
\hline $\begin{array}{l}\text { Imagen de la } \\
\text { Institución }\end{array}$ & & & & & & $x$ & & & & & & & & & & & & & $x$ \\
\hline
\end{tabular}

Fuente: literatura consultada.

Nota: Para la valoración del aporte de 18 autores consultados, se concentran 11 características en el estudio de la cultura organizacional. Se aprecian porcentajes en cada una de ellas: percepciones (44\%); creencias y expectativas (33\%); normas (39\%); ventaja competitiva (6\%); valores (56\%); símbolos (28\%); pensamientos/sentimientos (17\%); liderazgo/incentivos (39\%); relaciones/rituales (39\%); comunicación (11\%); visión y misión (11\%).

ISSN: 1390-9320, Vol. 5, No. 2, abril 2018 
La postura teórica de las autoras se sustenta principalmente en los aportes de Hofstede Geert (1991-2002) para definición de cultura y su desarrollo, y aportes de Edgar Schein (1986-2010) para la delimitación de algunas variables de cultura y la importancia del liderazgo como parte de la cultura.

La cultura organizacional refleja lo que la institución es al presente y su proyección futura, es su identidad y le permite diferenciarse de las demás. La cultura es considerada la personalidad de la organización y para su definición se sustenta en dos enfoques: el funcionalista y el psicodinámico.

1) Funcionalista por cuanto las personas son sociales por naturaleza, que hace que la cultura se estructure inexorablemente frente a la necesidad de supervivencia y adaptación al grupo por parte de sus miembros. Las personas necesitan sentirse parte del grupo, cubriendo así su necesidad de pertenencia e identidad.

2) Psicodinámica, porque la interacción con otros, implica compartir elementos y situaciones que dinamizan las relaciones por medio de sentimientos y pensamientos. El ser humano da significado a su entorno y crea significantes de sí mismo, de los otros y las vivencias compartidas al interior de la organización, éstas lo dinamizan internamente.

La cultura de una organización se desarrolla o rediseña. Es importante considerar la cultura y personalidad de cada integrante. Es decir, la cultura de la organización debe establecer características básicas, compartidas y asumidas por los miembros, pero sin despersonalizarlos y alienarlos. Esto implica, respetar principios culturales de las personas, siempre y cuando estas características, no sean irreconciliables con las de la organización.

Las autoras asumen seis variables, de aquellas expuestas por los autores referenciados, de las cuales (Visión- Misión y Valores) se integran y se las denomina filososfía institucional; luego imagen corporativa; liderazgo; comunicación; normas y reglamentos; y, relaciones. Los aspectos relacionados a lo simbólico, las percepciones y creencias, forman parte y estan implícitas en las otras variables. En la figura No2, se aprecia las dimensiones manifiesta y simbólica de las cuales se desagregan seis variables. 
Figura 2. Variables de la Cultura Organizacional para su valoración.

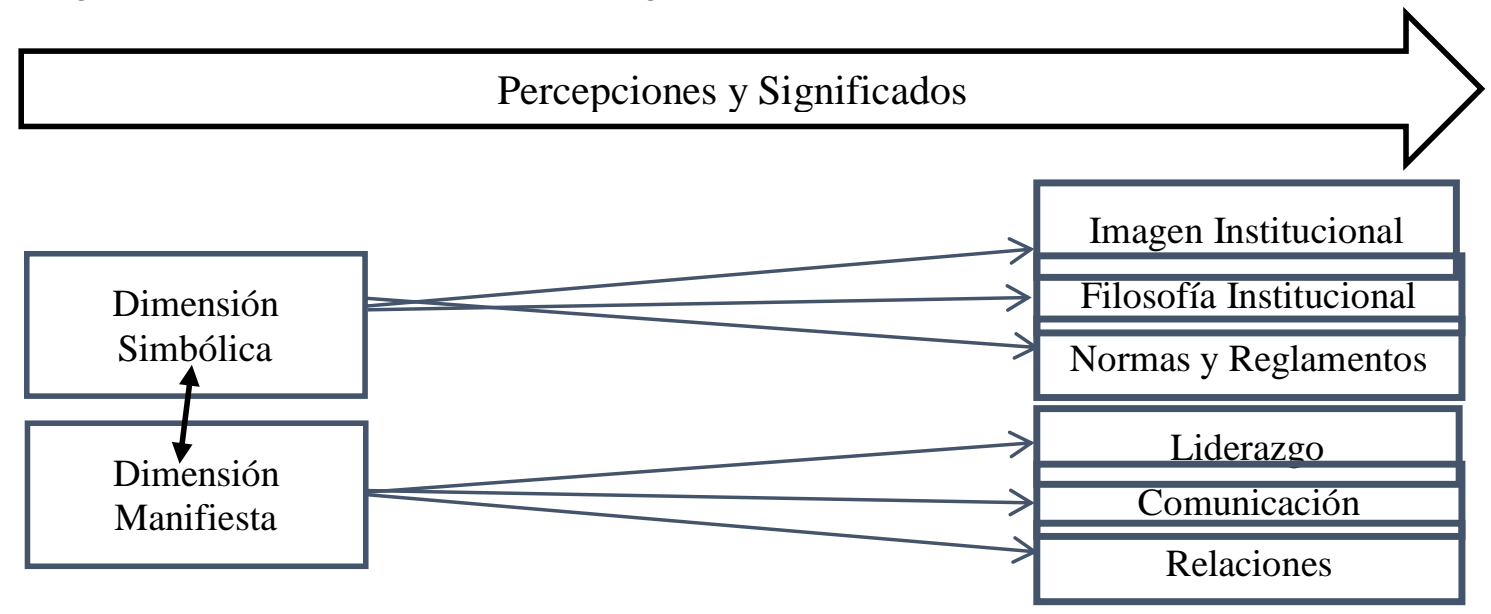

Fuente: Elaboración propia.

Dimensión simbólica. - Características que no se visualizan de primer momento, están del lado de lo simbólico y lo imaginario. Son pensamientos y sentimientos que experimentan los miembros de la organización, muchas veces no expresados abiertamente, encontrando formas explícitas o implícitas de manifestarse, pero que encierran un mensaje, un sentido compartido. Las variables de esta dimensión son:

1. Imagen Institucional, compuesta de símbolos percibidos en la relación con el entorno (forma de vestir; distribución de espacios, mobiliario; cuadros; frases; logotipos; carteleras); las historias; (origen; experiencias; anécdotas).

2. La Filosofía Institucional compuesta de Visión; Misión y valores, declarados por toda organización. Los símbolos relacionales son los valores manifestados en el lenguaje informal (bromas; palabras claves; rumores; especulaciones; siglas; sobre- nombres), la practica de valores universales (verdad; rectitud; solidaridad; pacificador) y los valores demandados por los directivos (puntualidad-retrasos; responsabilidad-negligencia; iniciativa-conformismo; lealtad-deslealtad; compromiso institucional-interés personal).

“Las compañías se basan en la ética, para elevar y medir continuamente los estándares de comportamiento corporativo. Aquellos que demuestran liderazgo en áreas como ciudadanía, integridad y transparencia, crean más valor para sus inversores, comunidades, clientes y empleados, y de esta manera fortalecen la ventaja comercial sostenible, explicó Timothy Erblich, Director Ejecutivo del Instituto Ethisphere". 
(Business Wire, 2016). Esto, referido al reconocimiento anual otorgado por el Instituto internacional de Ética, a las empresas más éticas en su gestión.

En cuanto a la Misión y Visión, reflejan la labor fundamental de toda organización al presente y el compromiso asumido a futuro con su entorno, por lo cual es necesario, "Crear una visión y compartirla con todo el personal y seguirla apasionadamente como lo han enfatizado Jack Welch, director general de General Electric; Helmut Maucher de Nestlé y otros importantes empresarios y directivos" (Siliceo, Casares, \& González, s.f.).

3. Las normas y reglamentos (también las políticas), proporcionan los lineamientos sobre la forma de hacer las cosas y los límites o condiciones al establecer las relaciones interpersonales y el cumplimiento de responsabilidades a todo nivel.

Dimensión manifiesta. - Las características de esta dimensión son observadas y escuchadas (percibidas), visibles en el diario vivir hacia el interior y exterior de la organización. Las variables de esta dimensión son:

4. Liderazgo. - Referido no solo a fundadores y directivos, sino también aquellos miembros que indistintamente de la posición que ocupan son referentes para otros, ya sea por su carisma, compañerismo, desempeño o ejemplo a imitar.

Para esta variable, se adoptan los cinco ámbitos planteados por Schein (1987) sobre liderazgo, referido a las percepciones; creencias y sentimientos que tienen los líderes respecto al grupo de personas a su cargo, en tanto que los estudios de liderazgo de Hofstede (2002) se relacionan con características culturales de los países donde funcionan las organizaciones. Las características sobre liderazgo planteadas por Schein (1987) son cinco:

- Percepción de los líderes en cuanto a la relación que tiene la institución con el entorno (dominar y cambiar el entorno; someterse al entorno y asumir lo impuesto; coexistir y estar en armonía con el entorno);

- Distribución de tareas de sus equipos de trabajo y manejo del tiempo, lo que se traduce en trabajo monocrónico (una tarea a la vez); trabajo policrónico (varias tareas a la vez). Los horarios; puntualidad o no; tiempo dedicado a reuniones;

- Percepción de la naturaleza humana: (buenos, malos, neutros; si son perfectibles o no; 
- Percepción sobre la naturaleza de la actividad humana (activos- pasivos; optimistaspesimistas; innovadores - conservadores; pueden ser controlados- manipulados);

- Naturaleza de las relaciones humanas: distribución del poder (competitivacooperativa- competente; individual- asociativa) y el cumplimiento de tareas y objetivos impulsado por (ley- autoridad o carisma). A partir de estas características, se van a definir cuatro tipos de liderazgo: integrativo; paternalista; exigente; apático.

5. Comunicación. - Caracterizada por la dinámica de las relaciones: la forma de comunicarse; procedimientos; sistemas de información; claridad en la comunicaciòn; coherencia entre lo comunicado y ejecutado e importancia de la información transmitida (relevante, de interés y utilidad para los miembros de la organización).

6. Las relaciones interpersonales, son fundamentales en toda organización, se dan a partir de encuentros (formales o informales); ceremonias formalidad o no; protocolos; nivel de participación e involucramiento de los miembros de los grupos que conforman la organización. En el caso de instituciones de educación superior, es importante que existan espacios para la recreación e integración de todos los miembros de la comunidad universitaria, especialmente estudiantes.

\section{CONCLUSIONES}

Las teorías y enfoques humanistas, permiten que autores como: Idalberto Chiavenato (2009); Sthephen Robbins (2013); Martha Alles (2007); Edgar Schein (1986- 2010); Geert Hosftede (1991), entre otros realicen estudios profundos sobre la cultura organizacional.

Las variables mayormente abordadas por los autores, resultan imprecisas y subjetivas al momento de valorarlas: percepciones- significados (44\%), creencias y expectativas (33\%); pensamientos y sentimientos (17\%); símbolos (28\%). Esto lleva a las autoras a la delimitación de variables objetivas.

Las autoras, luego del análisis de los estudios sobre cultura organizacional expuestos, desarrollan una conceptualización precisa de la cultura organizacional, integrando principios básicos de algunos autores prioritariamente Edgar Schein (1986-2010) y 
Geert Hofstede (1991), seguido enuncian dos dimensiones a partir de las cuales se desagregan seis variables que permiten la valoración de la cultura.

\section{REFERENCIAS BIBLIOGRÁFICAS}

Abravanel, H., Allaire, J., Firsirotu, M., Hobbs, B., Poupart, R., \& Simard, J. (2009). Cultura Organizacional. Colombia: Legis- Editores.

Alles, M. (2007). Comportamiento Organizacional. Buenos Aires: Granica.

Alles, M. (03 de 03 de 2016). Martha Alles Capital Humano. Obtenido de http://www.marthaalles.com/notas-rrhh-competitividad-y-gestion.php

Bobadilla, M., Callata, C., \& Caro, A. (2015). Compromiso laboral y cultura organizacional, el rol de la orientaciòn cultural en una empresa. Tesis para la optenciòn del grado científico de Magister en Desarrollo Organizacional y Direcciòn de empresas. Universidad del Pacifico.

Bohlander, G., Snell, S., \& Sherman, A. (2005). Administración de Recursos Humanos (Doceava ed.). México.

Bonavia, T., Prado, V., \& García, A. (2010). Adaptación al español del instrumento sobre cultura organizacional de Denison. SUMA Psicológica, 15-32.

Britez, V., Ortiz, A. M., \& Almazan, L. (2014). Metodología Aplicada en el estudio sobre las culturas organizacionales de las empreas Paraguayas del Alto. Aula de Encuentro, I(16), 121-137.

Carrillo, A. (2016). Medición de la Cultura Organizacional. Ciencias Administrativas, 61-73.

Chiavenato, I. (2009). Gestión del Talento Humano. En I. Chiavenato, Gestión del Talento Humano (pág. 10). México, D.F.: McGraw/Interamericana Editores S.A.

Codina, A. (2014). Habilidades Directivas. La Habana: Academia.

Contreras, C., Diaz, B., \& Hernández, E. (s.f.). Multiculturalidad: Su análisis y perspectivas a la luz de sus actores, clima. Eumed.

Cuesta, A. (2001). Gestión del Conocimiento, de la organización que aprende y de competencias: la era digital. Ingeniería Industrial, 3-8.

Cuesta, A. (2005). Tecnología de Gestión de Recursos Humanos (2da ed.). La Habana: Editorial Academia.

Cújar, A., Ramos, C., Hellman, H., \& Jorge, L. (2013). Cultura Organizacional: Evolución en la medición. Estudios Gerenciales, 7.

Esparza, A. (2009). Guía para la Ejecución del Programa de Cultura Institucional en la Administración Pública Federal. México: Instituto Nacional de las Mujeres. 
Felcman, I., Blutman, G., \& Méndez, M. S. (2001). Modelos de Cultura Organizacional en la Administración Pública Argentina. Centro de Investigaciónes en la Administración Pública Argentina.

Figueroa, L. (2015). Cultura Organizacional y Desempeño en una organización gubernamental. Tesis en optenciòn al título de licenciado en Psicologìa Industrial Organizacional. Guatemala: Universidad Rafael Landívar.

Globe Sari. (marzo de 2014). Obtenido de http://aptitudesprofesionales.blogspot.com/2014/03/el-liderazgo-culturalmodelo-globe.html

González, A., \& Fernández, E. (2000). La Cultura de la Organización en la Gestiòn de la Calidad Total. Ensaios e Ciencia, 99-114.

González, E., Fernández, M., \& Hernández, G. (julio a diciembre de 2013). Diagnóstico de la Cultura de la Calidad en una facultad universitaria. Estrategia y Gestión Universitaria, 1(1).

González, Y. (mayo de 2012). Cultura Organizacional en la empresa de seguros internacionales de Cuba. Trabajo de diploma. La Habana, Cuba: Universidad de la Habana.

Hellrieger, D., \& Slocum, J. (2003). Comportamiento Organizacional. (Décima). México: Thomson.

Hofstede, G. (1991). Culture and Organizations (Culturas y Organizaciones). New York: Mac Graw Hill.

Hofstede, G. (2002). Comportamiento y Desarrollo Organizacional. (E. Gainor, Recopilador) The Organization Development Institute International, Latinamerica.

Jones, G. (2015). Teoría Organizacional: Diseño y Cambio en las Organizaciones. México: Prentice Hall.

Katz, D., \& Kahn, R. (1966). The Social Psychology of organizations (La Psicología Social de las organizaciones). Wiley.

Las mejores practicas de RRHH en el mundo. (2011). Grandes Empleadores, 21-37.

Llanos, M. (2014). "La Cultura Universitaria para el desarrollo en la ciencia y tecnología en respuesta a las demandas sociales en Latinoamérica. Congreso Internacional "Tecnología, Universidad y Sociedad" (pág. 12). Santo DomingoRepública Dominicana: Universidad Central del Este.

Llanos, M. (2015). La Cultura: Estrategia en la Calidad del Servicio al Cliente. Madrid: Editorial Académica Española. 
Londoño, K. (2014). Estrategias de sensibilización para promover una cultura de calidad. Nueva Granada, 1-30.

Manjarrez, N. (2015). Gestión de Recursos Humanos con enfoque de Responsabilidad Social, en las Hosterías del sector turístico: caso cantón Pastaza. Tesis en opción al grado científico en Doctor en Ciencias Económicas. La Habana, Cuba: Universidad de la Habana.

Matías, B. (julio-septiembre de 2008). Modelo de Cultura de Calidad para la dirección de la formación del bachiller. Ciencia UANL, 11(3).

Morelos, J., \& Fontalvo, M. (Enero-junio de 2014). Análisis de los factores determinantes de la Cultura Organizacional en el Ambiente Empresarial. Entramado, 10(1), 96-105.

Ogliatri, E., McMillen, C., Altschul, C., Arias, M. E., Bustamante, C., Dávila, C., . . . Martínez, S. (1999). Cultura y liderzgo organizacional en 10 países de América Latina. El estudio Globe. Academia, Revistra Latioamericana de Administración, 28-57.

Ortiz, E. (2010). Los índices culturales de Hofstede y su impacto en los estilos de liderazgo en empresas extranjeras que operan en Puerto Rico. Forum Empresarial, 59-81.

Ortiz, P., \& Olaz, A. (2015). Propuesta de un Modelo de Análisis de Cultura y Clima Laboral. 1-15.

Ospina, D. (2014). ¿Por què un modelo de Cultura Organizacional? Tesis para la opciòn al título de especialidad en Gestiòn de Desarrollo Administrativo. Colombia: Universidad Militar Nueva Granada.

Peralta, M. C. (2005). Descripción de la Cultura Organizacional y los Valores de convivencia y responsabilidad social en un Call Center de Bogotá. Psicología desde el Caribe, 51-73.

Pérez, M. (2008). Test de Kim Cameron para diagnosticar la Cultura Organizacional. Material de la Cátedra de Administración de Personal- Facultad de Ciencias Económicas. Buenos Aires: Universidad de Buenos Aires.

Piva, A. M. (2006). Cultura Organizacional: Un caso de análisis. Título para la optenciòn de grado de licenciado en Sociologìa. Argentina: Facultad de Humanidades y Ciencias de la Educación.

Pujol, L., Foutel, M., \& Musticchio, A. (2014). Hacia la Construcciòn de un modelo para el abordaje empírico de la identidad y la cultrua organizacional: Resultados preliminares. Argentina: Universidad Nacional del Mar del Plata. 
Quiroz, T., \& Valtierrez, D. (2010). Dimensiones Culturales en la empresa estatal de Autoservicios sucursal Delicias Chiguagua. Revista Mexicana Agroindustrial, 27(Quinta Epoca), 375-386.

Robbins, S., \& Coulter, M. (2005). Administración (Octava ed.). México: Pearson.

Robbins, S., \& Judge, T. (2013). Comportamiento Organizacional (Décimo quinta ed.). México: Pearson.

Rodriguez, C. (2011). Cultura Organizacional.

Rodriguez, R. (2009). La Cultura Organizacional un potencial activo estratégico desde la perspectiva de la administración. Invenio, 67-92.

Salazar, A. M. (octubre de 2008). Estudio de la Cultura Organizacional, según Cameron y Quinn: Caso de una empresa del sector asegurador Venezolano. Tesis de Especialidad. Venezuela: Universidad Católica Andrés Bello.

Sánchez, I. (2010). Desarrollo de un instrumento de medida de la cultura organizativa: Un modelo confirmativo en los hoteles españoles. Revista Europea de Dirección y Economía de la Empresa, 19(1), 107-126.

Schein, E. (1986). What do you need know about Organization Culture (Qué necesitas saber acerca de Cultura Organizacional). Training and Devopment Journal, 3036.

Schein, E. (1987). La Cultura Empresarial y el Liderazgo. Barcelona.

Schein, E. (1993). Legitimating Clinical Research in the Study of Orgnization Culture, (Investigaciones clínicas en el Estudio de la Cultura Orgnizacional). Journal of Counseling and Development, 71, 703- 708.

Schein, E. (1996). Cuture: The missing concept in Organization Study (Cultura: El concepto perdido en el estudio de la organización). Administrative Science Quarterly, 229, 229-240.

Schein, E. (2010). The role of organization development in the human resource function (El rol del desarrollo organizacional en la función de los recursos humanos). OD Practitioner, 42(4).

Siliceo, A., Casares, D., \& González, J. L. (s.f.). Liderazgo, valores y cultura organizacional: Hacia una organización competitiva (Primera ed.). Mac Graw Hill.

Solomon, M. R. (2013). Comportamiento del Consumidor. México: Pearson.

Sthephen, R., \& Judge, T. (2009). Comportamiento Organizacional. México: PearsonPrentice Hall.

Thomás, M., \& Ion, G. (2008). Contrastando dos modelos de análisis de la cultura organizativa de la universidad. Bordón, 131-147. 
Vargas, J. (2007). La Culturocracia Organizacional en México. México: Eumed.

Velasquez, M. (2012). Etica en los Negocios. México: Pearson.

Welch, J. (2004). El Liderazgo. México: Mac Graw Hill. 